\title{
LIPID STAINING ON SEMITHIN SEGTIONS WITH SUDAN BLAGK B OR NILE BLUE SULPHATE. APPLICATION TO INTESTINAL FAT ABSORPTION
}

\author{
Marie-France SIRE et Jean-Marie Vernier \\ Laboratoire de Biologie-Vertébrés, Université Paris-Sud, \\ 91405-ORSAY, France
}

Received for publication August 16, 1979 and in revised form September 18, 1979

\begin{abstract}
The specific characterization of lipids was investigated by lysochromes* on routine semithin sections of osmium fixed tissues embedded in epon 812 for ultrastructural study. The work was done on trout and rat intestine during fat absorption. The best results were obtained after prefixation with paraformaldehyde.

1-1.5 $\mu \mathrm{m}$ sections were stained by Sudan black $B$ and Nile blue sulphate under well defined conditions. The use of $\mathrm{H}_{2} \mathrm{O}_{2}$ makes staining by lysochromes possible because: (i)-it eliminates the primary blackness due to osmium fixation and allows the visualisation of lysochromes which are dissolved in the lipids; (ii) -it increases the porosity of resin and allows the excess of lysochromes into the tissue lipids. The results obtained with Nile blue sulphate (colouring blue and red lysochrome) are discussed. A counterstaining with Ehrlich hematoxylin can be performed for later morphological study.
\end{abstract}

A further ultrastructural study allowed the identification of stained structures by Sudan black $B$ and Nile blue sulphate.

Les colorants usuels des lipides, sulfate de bleu de Nil et noir Soudan B, n'ont pu jusqu'ici être utilisés avec succès sur coupes semi-fines de tissu inclus dans les résines en particulier l'épon. Pour cette raison, Snipes (12) préconise l'emploi de la $p$-phenylènediamine, la reaction étant probablement basée sur l'oxydation du colorant par les lipides osmiés $(3,7)$. La mise en évidence des lipides lors de l'étude ultrastructurale ultérieure du tissu repose sur une méthode qui n'est pas davantage spécifique, la méthode OTO de Seligman et al. (11). A aucun moment d'une étude morphologique concernant les lipides tissulaires, l'expérimentateur ne bénéficie donc d'une méthode sûre de caractérisation.

Notre réussite antérieure (15) pour caractériser les glucides sur coupes semifines de tissu inclus dans l'épon par utilisation de l'A.P.S. (confirmant la réaction au protéinate d'Ag. de l'étude ultrastructurale) nous a permis de mettre au point une technique de coloration des coupes semi-fines par le noir Soudan et le bleu de Nil. L'étude porte sur la muqueuse intestinale en phase d'absorption des lipides alimentaires.

* lysochrome: substance which can dissolve into cellular constituents which stain them. Lysochrome: substance capable de colorer les constituants cellulaires en s'y dissolvant (lyso: dissolution, chrome: couleur). 


\section{MATERIEL ET METHODES}

\section{Prélèvement, fixation et inclusion des échantillons}

a) Muqueuse intestinale de Rat.

Nous avons reproduit les conditions expérimentales de Friedman et Cardell (4). Des rats de $250 \mathrm{~g}$ sont mis a jeûner $24 \mathrm{~h}$. Âpres une légère anesthésie ils sont gavés avec $1.5 \mathrm{ml}$ d'huile de maïs. $1 \mathrm{hr}$ après le gavage des segments d'intestin situés à $15 \mathrm{~cm}$ du pylore sont prélevés et fixés a $4^{\circ} \mathrm{C}$ pendant $2 \mathrm{hr}$ par le glutaraldéhyde a $3 \%$ dans du tampon phosphate $0.1 \mathrm{M}$ a $\mathrm{pH}$ 7.3. Après lavage dans du tampon phosphate sucré $(5 \%)$ et postfixation pendant $2 \mathrm{hr}$ par le tétroxyde d'osmium à $2 \%$ tamponné par le phosphate $0,1 \mathrm{M}$ à $\mathrm{pH} 7.3$ (concentration finale $1 \%$ ), les échantillons sont deshydratés et inclus dans l'épon 812.

b) Muqueuse intestinale de Truite.

Les échantillons sont préleves sur des animaux normalement alimentés (1 repas matinal, ad lib.) $4 \mathrm{hr}$ après la prise de nourriture (formation de particules lipidiques $<1000 \AA$, VLDL); sur des animaux préalablement mis à jeûner durant 8 jours et réalimentés (la prise de nourriture est alors très importante), 24 hr après le repas (formation de particules lipidiques de 600 a $3500 \AA$ de diamètre, VLDL et chylomicrons); et sur des animaux ayant ingéré $400 \mu \mathrm{l}$ d'acide linoléique, $13 \mathrm{hr}$ après le gavage (formation de grosses gouttelettes lipidiques de 1 à $3 \mu$ de diamètre dans le cytosol.

Ils sont fixés à $4^{\circ} \mathrm{C}$ pendant $15 \mathrm{mn}$ par le paraformaldéhyde à $4 \%$ dans du tampon cacodylate de sodium $0.15 \mathrm{M}$ à pH 7.3. Rapidement lavés dans le tampon et postfixés pendant $1 \mathrm{hr}$ par le tétroxyde d'osmium $2 \%$ dans le tampon cacodylate

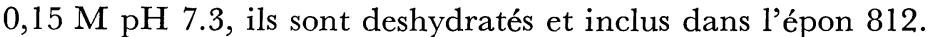

c) Pour la mise en évidence ultrastructurale des lipides par la méthode OTO, les échantillons sont fixés à $4^{\circ} \mathrm{C}$ pendant $2 \mathrm{hr}$ par le tétroxyde d'osmium $2 \%$ dans du tampon cacodylate de sodium $0.15 \mathrm{M}, \mathrm{pH} 7.3$, déshydrates et inclus dans l'epon 812.

\section{Coloration par le noir Soudan et le bleu de Nil}

Les coupes semi-fines de 1 a 1,5 $\mu$ sont montées sur lames de verre.

a) Coloration par le noir Soudan B.

Les coupes sont traitées comme suit: solution aqueuse d' $\mathrm{H}_{2} \mathrm{O}_{2}$ à $5 \%, 20 \mathrm{mn}$; eau distillée, $5 \mathrm{mn}$; solution alcoolique saturée de noir Soudan $\mathrm{B}, 45 \mathrm{mn}$ à $60^{\circ} \mathrm{C}$; alcool $70^{\circ}, 30 \mathrm{sec}$; eau courante, $30 \mathrm{sec}$. Les coupes sont séchées, puis observées sans milieu de montage ou après montage dans le baume du Canada.

b) Coloration par le sulfate de bleu de Nil.

Les coupes sont traitees comme suit: solution aqueuse d' $\mathrm{H}_{2} \mathrm{O}_{2}$ à $5 \%, 20 \mathrm{mn}$; eau distillée, $5 \mathrm{mn}$; solution aqueuse de sulfate de bleu de Nil à $1 \%, 20 \mathrm{mn}$ à $60^{\circ} \mathrm{C}$; lavage rapide par l'eau distillée à $60^{\circ} \mathrm{C}$; solution d'acide acétique à $1 \%, 30 \mathrm{sec}$ à $60^{\circ} \mathrm{C}$; eau courante, $30 \mathrm{sec}$. Les coupes sont sechées, puis observées sans milieu de montage ou après montage dans le baume du Canada.

3. Etude ultrastructurale

a) Les coupes fines sont contrastées par l'acétate d'uranyle et le citrate de plomb. 


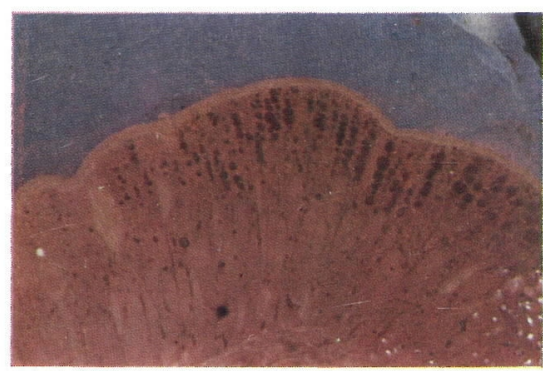

FIG. 1.

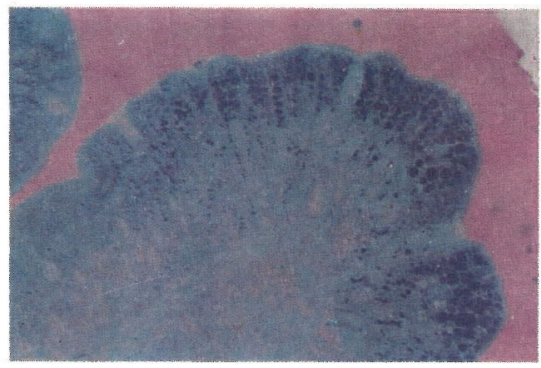

FIG. 3.

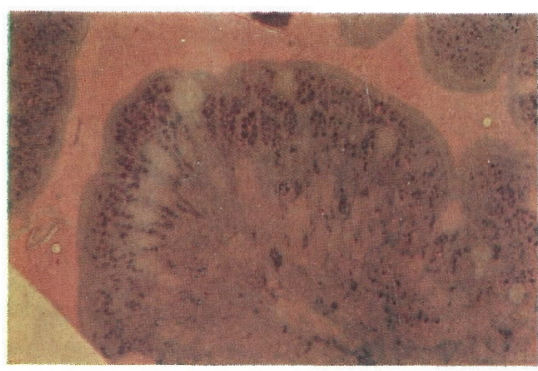

FIG. 5 .

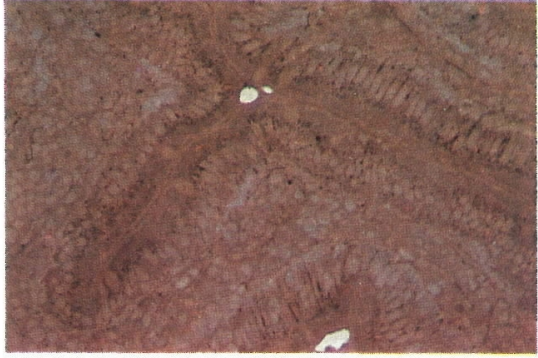

FIG. 2.

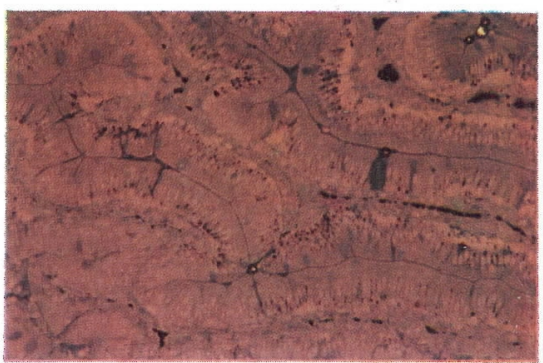

FIG. 4.

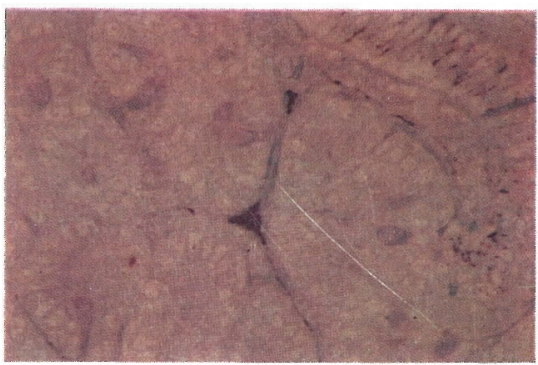

FIG. 6.

FIG. 1 a 6. - Cioupes semi-fines de tissu inclus dans l'épon. Coloration par le noir Soudan (fig. 1 et 2) et le bleu de Nil (fig. 3 a 6 ).

FIG. 1. Epithelium intestinal de Truite $13 \mathrm{hr}$ après ingestion d'acide linoléique. De grandes gouttelettes soudanophiles apparaissent dans le cytoplasme des entérocytes. $\times 190$ en 24/36, immersion

FIG. 2. Muqueuse intestinale de Rat après ingestion d'huile de Maïs. Des granules positifs sont visibles dans le hyaloplasme des entérocytes, les espaces intercellulaires et dans la lamina propria. $\times 100$ en $24 / 36$

Frg. 3. Mêmes conditions que pour la figure 1. La coloration bleue obtenue témoigne du caractère acide des gouttelettes lipidiques. $\times 160$ en $24 / 36$

FIG. 4 et 6 . Mêmes conditions que pour la figure 2.

Fig. 4. Les granules soudanophiles de la figure 2 sont ici colorés en rouge (lipides neutres essentiellement). $\quad \times 60$ en $24 / 36$

Fig. 5. Muqueuse intestinale de Truite à jeun depuis 8 jours et réalimentée. $24 \mathrm{hr}$ après le repas, le hyaloplasme des cellules montre des structures colorées de tailles variées. $\quad \times 80$ en 24/36

Frg. 6. Détail montrant les granules dans la lamina propria sous-epithéliale. $\quad \times 100$ en $24 / 36$ 
b) Pour la mise en evidence des lipides selon la méthode OTO de Seligman et al. (11), les coupes sont recueillies sur grilles en or et placées succéssivement sur une solution aqueuse de thiocarbohydrazide à $1 \%, 1 \mathrm{hr}$ à $50^{\circ} \mathrm{C}$ et sur une solution aqueuse de tetroxyde d'osmium à $2 \%, 1 \mathrm{hr}$ à $50^{\circ} \mathrm{C}$.

\section{RESULTATS}

Les figures 1 et 2 montrent des exemples de coloration obtenues avec le noir Soudan chez la Truite et le Rat.

Après coloration par le bleu de Nil, les enterocytes et la lamina propria sous épithéliale contiennent des granules rouges de tailles variées (figures 4,5 et 6 ). Les grosses gouttelettes soudanophiles obtenues après ingestion d'acide linoléique (figure 1) apparasisent colorées en bleu quelles que soient les modifications apportées au protocole expérimental (dilution des solutions de bleu de Nil et augmentation du temps de coloration...).

Les techniques de fixation utilisées permettent toutes une bonne étude ultrastructurale (figures 7 et 9, paraformaldéhyde-osmium; figure 10, glutaraldéhydeosmium; figure 8, osmium seul). Le paraformaldéhyde donne d'excellents résultats tandis qu'après préfixation par le glutaraldéhyde des granules lipidiques presentent quelques figures d'extraction.

L'étude ultrastructurale permet l'identification des structures colorées par le noir Soudan et le bleu de Nil. Chez le Rat, les granules contenus dans les cisternae $\mathrm{du}$ reticulum endoplasmique et les vésicules golgiennes (figure 10) ont une taille comprise entre 2000 et $10,000 \AA$ (chylomicrons) et peuvent donc pour la plupart être observés apres utilisation des deux colorants. Chez la Truite, la situation est très différente. Dans des conditions normales d'alimentation (figures 7 et 8) seuls se forment des granules de taille inférieure à $1500 \AA$, le plus souvent comprise entre 600 et $800 \AA$ (VLDL). Le noir Soudan et le bleu de Nil ne permettent alors que la matérialisation des amas de granules situés dans certaines vésicules golgiennes, dans les espaces intercellulaires et dans les espaces interstitiels de la lamina propria. A ces structures s'ajoutent, chez la Truite qui absorbe massivement après un jeûne, les granules dont la taille atteint celle de petits chylomicrons contenus dans les éléments apicaux du réticulum et dans les éléments golgiens ainsi que des gouttelettes lipidiques non limitées par une membrane apparues dans le cytosol.

Une double étude en microscopie optique et en microscopie électronique est donc indispensable pour la caractérisation des lipides au cours de l'absorption intestinale.

\section{DISGUSSION}

La mise en évidence au moyen de -lysochromes représente la plus simple et la plus efficace des méthodes de détection des lipides auxquels ils confèrent une teinte due uniquement à leur dissolution. Ce phénomène physique simple ("coloration") ne se produit pas si le lipide, quelle que soit sa nature, est à l'état solide. Les coupes utilisées sont obtenues au microtome à congélation $(1,5)$.

L'application aux coupes semi-fines de tissu inclus dans l'péon ou autres résines souléve de nombreuses difficultés et l'utilisation du noir Soudan et du bleu de Nil 


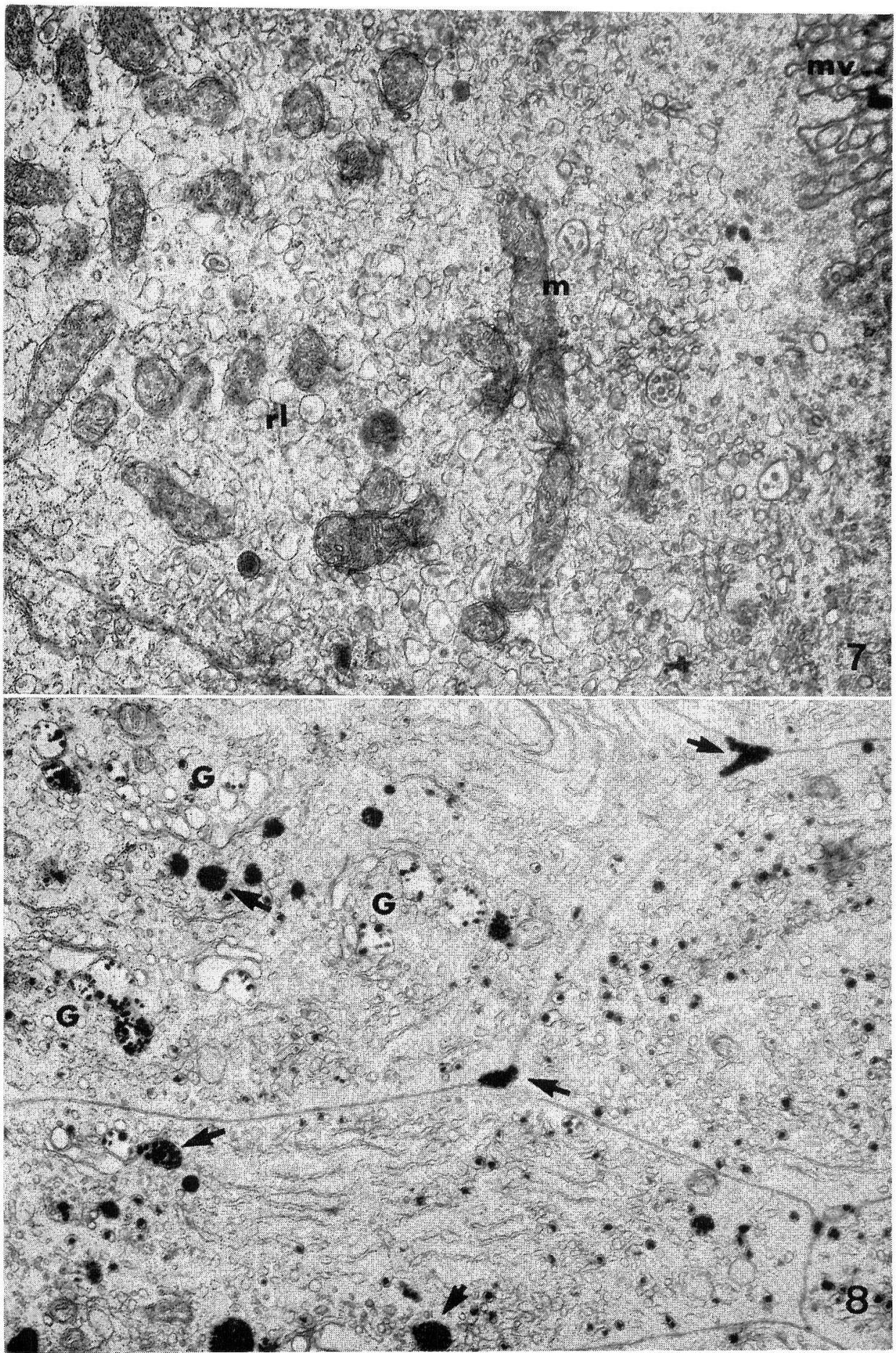




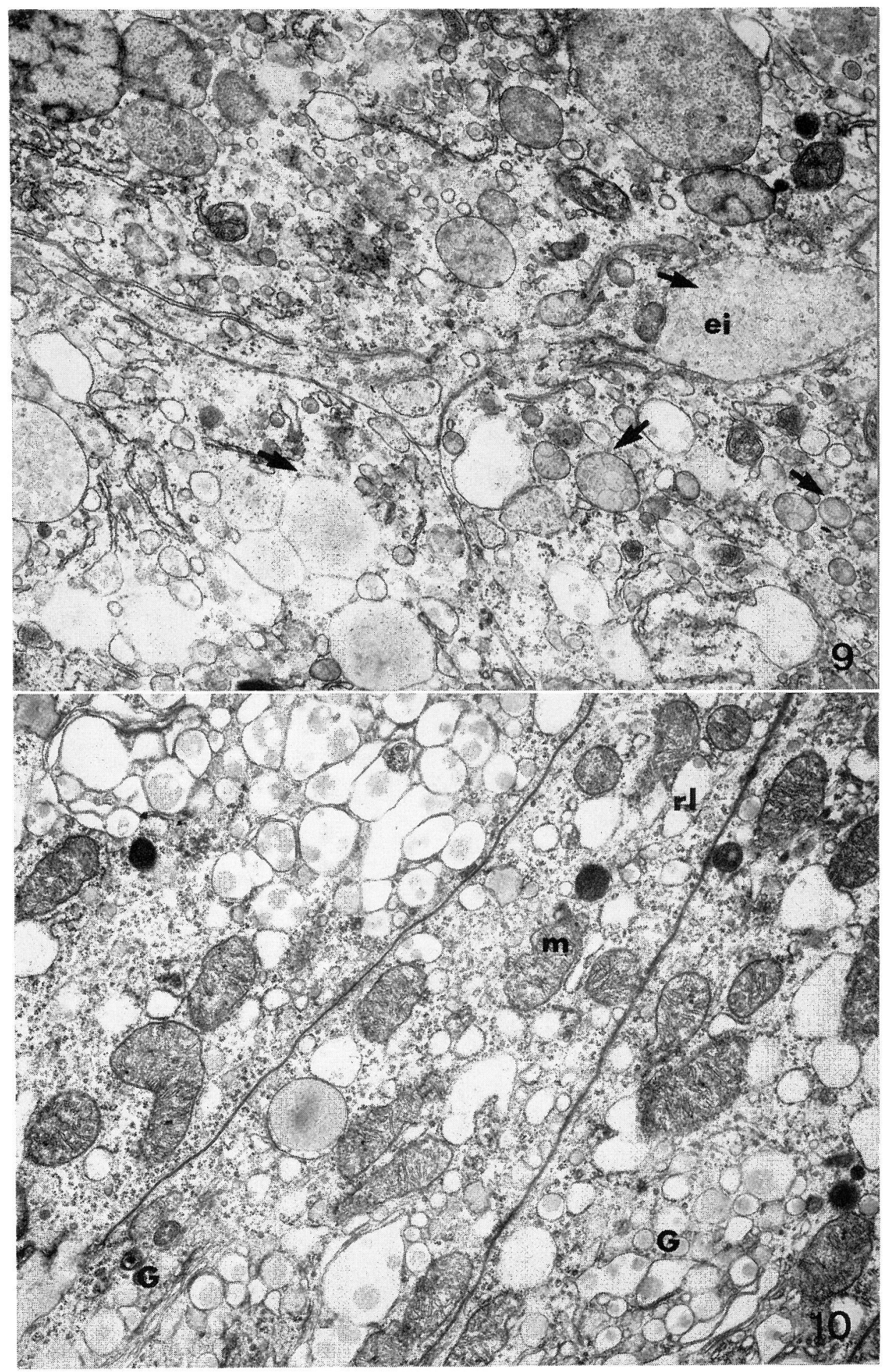


(en fait son composant lysochrome, le rouge de Nil) n'a pas jusqu'ici été couronnée de succès. Pour cette raison l'emploi de la p-phénylènediamine initialement proné pour la détection des groupements aldéhydes dont on utilise les propriétés peroxydasiques est actuellement préconisé $(7,12,13)$. Le premier temps de cette méthode repose sur la réaction du tetroxyde d'osmium qui forme un complexe avec les lipides $(11,14)$. Cette réaction, utilisée depuis fort longtemps pour la détection des lipides (noircissement osmique primaire) et qui va de pair avec leur insolubilisation, n'est malheureusement pas spécifique puisqu'elle n'est pas une propriété générale des lipides ni l'apanage de ce groupe de composés. Dans le deuxième temps, le tetroxyde d'osmium combiné se comporte comme un peroxyde vis à vis de la pphénylènediamine, mais la réaction n'en devient pas pour autant spécifique.

Pour la caractérisation ultrastructurale des lipides, la méthode OTO repose également sur la réaction avec les lipides du tetroxyde d'osmium utilisé pour la fixation du tissu. Leur contraste est ensuite amplifié par un nouveau noircissement osmique, le thiocarbohydrazide, apporté en excès, servant de liant entre les deux couches d'osmium. Cette methode n'est donc pas davantage spécifique, les éléments tissulaires les plus contrastés sont ceux détenant le plus d'osmium donc en principe les lipides. L'extension de l'usage des lysochromes aux coupes semi-fines de tissu inclus dans l'epon et fixé par le tetroxyde d'osmium pour une étude ultrastructurale ulterieure constitue donc un apport fondamental.

L'utilisation d' $\mathrm{H}_{2} \mathrm{O}_{2}$ permet de remplir deux conditions preliminaires fondamentales indispensables à l'emploi des lysochromes:

—elle élimine le "noircissement" primaire du à la fixation osmiée et qui sert de support aux réactions signalétiques des lipides utilisées jusqu'ici. Le "blanchiment" des coupes jusqu'à la décoloration totale peut être suivi au microscope. Cette technique du blanchiment était utilisée classiquement pour vérifier l'insolubili-

Fig. 7 et 8 . Entérocytes de Truite normalement alimentée, $4 \mathrm{hr}$ après la prise de nourriture.

Fig. 7. Zone apicale d'un entérocyte. Acétate d'uranyle-citrate de plomb.

Les cavités du réticulum lisse contiennent des granules dont le diamètre ne dépasse pas $800 \AA$. $\times 18,000$

Fig. 8. Zone golgienne de quelques entérocytes. Méthode, OTO.

On retrouve contrastés par l'osmium, les granules du réticulum mais aussi les granules contenus par divers éléments golgiens. Seuls seront matérialisés par les lysochromes les granules accumulés dans certaines vésicules golgiennes et les espaces intercellulaires (fléches). L'ensemble des granules est assimilable à des VLDL. $\times 13,000$

Fig. 9. Entérocytes de Truite dans les mémes conditions que la figure 5. Acétate d'uranyle-citrate de plomb.

Dans ces conditions de surcharge alimentaire, réticulum endoplasmique et éléments golgiens contiennent des granules de 600 à $3,000 \AA$ A. Les plus volumineux d'entre eux, ceux accumulés dans diverses vséicules golgiennes et lés espaces intercellulaires, ainsi que les gouttelettes du cytosol (flèches) sont détectables par les lysochromes. $\quad \times 16,000$

Fig. 10. Entérocytes de Rat, zone des mitochondries et zone golgienne, dans les mêmes conditions que les figures 2,4 et 6 . Acétate d'uranyle-citrate de plomb.

Les cavités du réticulum ainsi que les vésicules golgiennes contiennent des granules de 2,000 a $10,000 \AA$. La plupart d'entre eux sont matérialisables par les lysochromes. Ils correspondent à des chylomicrons. $\times 12,000$
e.i.: espace intercellulaire
m.v.: microvillosités
G: Golgi
m.: mitochondries
r.l.: réticulum lisse 
sation des lipides tissulaires par le tetroxyde d'osmium. Après traitement du tissu osmié par le benzène, le chloroforme, le toluène ou le xylène, retour á l'eau et blanchiment par l'eau oxygénée, les lipides sont encore présents et peuvent donc être colorés par le noir Soudan B. Si l'on traite par le solvant, non avant, mais aprés blanchiment par l'eau oxygénée, il dissout les lipides et le noir Soudan B ne colore plus rien (8). L'élimination du noircissement osmique permet la visualisation du lysochrome dissout dans les lipides.

-elle augmente la porosité de la résine $(9,10)$ et permet l'accès du lysochrome aux lipides tissulaires. Aucune de ces deux conditions n'est remplie dans la tentative de Mc Gee-Russell et Smale (6).

Le noir Soudan B est le lysochrome le plus efficace pour caractériser les lipides. Le bleu de Nil est un colorant qui contient une impureté, le rouge de Nil, base très faible dont les sels sont entièrement hydrolysés par l'eau; la base libre existe donc dans les solutions aqueuses. Cette base est un lysochrome et, à ce titre, confére une teinte rose à tous les lipides. Cette teinte est masquée ou modifiée par la présence de lipides acides colorés en bleu par le bleu de Nil, colorant qui ne prend pas sur les lipides neutres.

Dans le cas de l'absorption intestinale, les teintes obtenues varient du rose au rouge vineux. Les variations tiennent à la nature complexe des lipides ou des lipoprotéines matérialisés dont les triglycérides forment le constituant essentiel. Cette coloration a la même signification que toutes les "colorations" par des lysochromes et permet d'affirmer la présence de lipides dans les structures ainsi mises en évidence. Dans le cas des gouttelettes bleues présentes dans le cytosol des entérocytes de Truite traitées par l'acide linoléique (fig. 3), l'interprétation est moins immédiate. La coloration bleue n'indique que la presence de groupements électronégatifs; le bleu de Nil est un colorant basique et met en évidence tous les groupements acides, lipidiques ou non. Le résultat positif obtenu avec le noir Soudan (fig. 1) permet toutefois de conclure que ces gouttelettes sont lipidiques. Exceptionnellement, il semblerait que ces gouttelettes de lipides acides puissent correspondre à de l'acide linoléique temporairement stocké dans le cytosol.

La meilleure préservation des lipides a été obtenue après préfixation par le paraformaldehyde. Ceci vient conforter le fait que les meilleurs resultats pour les coupes à congelation ont été obtenus après fixation plus ou moins prolongée par le formol.

L'utilisation d' $\mathrm{H}_{2} \mathrm{O}_{2}$ permet d'obtenir la libération des groupements phosphates des acides nucléiques, bloqués par l'osmium de la fixation (2). Une coloration nucléaire est donc possible par une laque à condition d'éviter les laques aluminiques. L'hématoxyline de Ehrlich donne de bons resultats.

\section{BIBLIOGRAPHIE}

1. Bergot, P. and Flechon, J. E.: Forme et voie d'absorption intestinale des acides gras à chaine longue chez la Truite arc-en-ciel (Salmo gairdneri Rich.). II. Lipides "étalés". Ann. Biol. anim. Bioch. Biophys. 10; 473, 1970.

2. Cooley, C. A., Lucas, J. A. and Schardein, J. L.: A modified hematoxylin-safranin stain for 0,5-2 $\mu$ sections. Stain Technol. 47; 44, 1972.

3. Estable-Puig, J. F. and Blumberg, J. M.: Paraphenylediamine staining of osmium fixed, plasticembedded tissue for light and phase microscopy. J. Neuropath. exp. Neurol. 24; 531, 1965. 
4. Friedman, H. I. and Cardell, R. R.: Alterations in the endoplasmic reticulum and Golgi complex of intestinal epithelial cells during fat absorption and after termination of this process: a morphological and morphometric study. Anat. Rec. 188; 77, 1977.

5. Garcia-Segura, L. M., Martinez-Rodriguez, R., Toledano, A., Dia-Gonzales, P., De Augustin, M. and Gamonal, A.: A histochemical research of phospholipids and simple triglyceride esters in human liver tissues. Ann. Histochim. 20; 237, 1975.

6. McGee-Russell, S. M. and Smale, N. B.: On colouring epon-embedded tissue sections with Sudan black B or Nile blue A for light microscopy. Quart. J. micr. Sci. 104; 109, 1963.

7. Korneliussen, H.: Identification of muscle fiber types in "semi-thin" sections stained with p-phenylenediamine. Histochemie 32; 95, 1972.

8. Lison, L.: Histochimie et Cytochimie Animales; Principes et Méthodes. Gauthier-Villars, Paris, 1960.

9. Poo, C. R.: Hematoxylin-eosin staining of $\mathrm{OsO}_{4}$-fixed epon-embedded tissue; prestaining oxidation by acidified $\mathrm{H}_{2} \mathrm{O}_{2}$. Stain Technol. 44; 75, 1969.

10. Pool, C. R.: Prestaining oxidation by acidified $\mathrm{H}_{2} \mathrm{O}_{2}$ for revealing Schiff-positive sites in eponembedded sections. Stain Technol. 48; 123, 1973.

11. Seligman, A. M., Wasserkrug, H. L. and Hanker, J. S.: A new staining method (OTO) for enhancing contrast of lipid-containing membranes for droplets in osmium tetroxide-fixed tissue with osmiophilic thiocarbohydrazide (T.C.H.). J. Cell Biol. 30; 424, 1966.

12. Snipes, R. L.: Identification of lipids for intestinal absorption studies in resin-embedded tissue. Microsc. Acta 79; 127, 1977a.

13. Snipes, R. L.: Limited fat absorption in the large intestine of mice. A morphological study. Acta Anat. 99; 435, 1977b.

14. Stoeckenius, W. and Mahl, S. G.: Studies on the reaction of osmium tetroxide with lipids and related compounds. Lab. Invest. 14; 1198, 1965.

15. Vernier, J. M. and Sire, M.F.: Dosage du glycogène hépatique sur coupes à l'Epon. Evaluation quantitative du glycogène hépatique par histophotométrie, sur coupes semi-fines de tissu inclus dans 1'Epon. Acta histochem. cytochem. 9; 1, 1976. 